\title{
Inhibition of focal adhesion kinase induces apoptosis in bladder cancer cells via Src and the phosphatidylinositol 3-kinase/Akt pathway
}

\author{
DEBO KONG ${ }^{1}$, FENG CHEN $^{2}$ and NI SIMA ${ }^{3}$ \\ ${ }^{1}$ Department of Urology, The First Affiliated Hospital, School of Medicine, Zhejiang University, Hangzhou, \\ Zhejiang 310003; ${ }^{2}$ Department of Surgery, The Jiujiang Traditional Chinese Medicine Hospital, Jiujiang, \\ Jiangxi 332000; ${ }^{3}$ Department of Gynecologic Oncology, Women's Reproductive Health Key Laboratory of Zhejiang, \\ Women's Hospital, School of Medicine, Zhejiang University, Hangzhou, Zhejiang 310003, P.R. China
}

Received March 4, 2015; Accepted August 3, 2015

DOI: $10.3892 /$ etm.2015.2745

\begin{abstract}
Focal adhesion kinase (FAK) is a $125-\mathrm{kDa}$, cytosolic, non-receptor, protein tyrosine kinase localized at focal adhesions that can be activated by multiple inputs and in different manners. FAK is implicated in signaling pathways regulating cell movement, invasion, survival, gene expression and cancer stem cell self-renewal. The aim of the present study was to investigate whether FAK plays a role in the apoptosis of bladder cancer cells. The study employed in situ deoxynucleotidyl transferase-mediated dUTP-biotin nick end labeling and Annexin V labeling flow cytometry. It was found that both the knockdown of FAK and the suppression of FAK phosphorylation were able to induce apoptosis in bladder cancer cells. Caspase-3 was activated during the apoptosis induced by the suppression of FAK phosphorylation. Src was involved in FAK-regulated apoptosis in bladder cancer cells, while the suppression of Src phosphorylation was able to inhibit FAK tyrosine phosphorylation and induce apoptosis. Furthermore, phosphatidylinositol 3-kinase (PI3K)/Akt signaling was inhibited via the suppression of FAK tyrosine phosphorylation. Conversely, the expression of neither the general nor the tyrosine-phosphorylated FAK was regulated by inhibiting PI3K/Akt, which suggested that PI3K/Akt acted downstream of FAK to regulate apoptosis in bladder cancer cells. These findings indicate the presence of a mechanism of apoptosis involving FAK-mediated oncogenic signaling. FAK may function as an important regulator of extracellular
\end{abstract}

Correspondence to: Dr Ni Sima, Department of Gynecologic Oncology, Women's Reproductive Health Key Laboratory of Zhejiang, Women's Hospital, School of Medicine, Zhejiang University, Building 1, 2 Xueshi Road, Hangzhou, Zhejiang 310003, P.R. China

E-mail: simani@163.com

Key words: focal adhesion kinase, apoptosis, bladder cancer, Src, phosphatidylinositol 3-kinase/Akt signaling-mediated apoptosis in bladder cancer and be used as a novel therapeutic target in the treatment of the condition.

\section{Introduction}

Bladder cancer is the second most common genitourinary cancer and the eleventh most common malignancy worldwide. It has been estimated that $\sim 429,800$ new cases of bladder cancer and 165,100 bladder cancer-related mortalities occurred in 2012 worldwide, accounting for $3 \%$ of the total new cancer cases (1). Furthermore, bladder cancer is now the most frequent cancer of the urinary tract and the seventh most frequent malignancy in men worldwide. An increasing trend in the incidence and mortality rates of bladder cancer has been noted in the past 30 years (2). Among all newly diagnosed cases, $75 \%$ of patients exhibit a non-muscle-invasive tumor without invasion into bladder detrusor; however, $25 \%$ of patients present with muscle-invasive bladder tumors, which means that the bladder detrusor has been invaded by the cancer (3). Furthermore, between 50 and $70 \%$ of the cases of non-muscle-invasive tumors will recur following transurethral resection, despite intravesical chemotherapy or Bacillus Calmette-Guérin immunotherapy, and 10-20\% will progress to muscle-invasive bladder tumors in 5 years (4). Notably, lymph node metastases are confirmed by pathological examination in $25 \%$ of patients with muscle-invasive cancer who have undergone radical cystectomy, while it is estimated that one-third of patients with muscle-invasive cancer have undetected metastases at first diagnosis (5). There is, therefore, an active interest in the study of the molecular mechanism of bladder cancer.

Focal adhesion kinase (FAK) is a $125-\mathrm{kDa}$ tyrosine kinase found in focal adhesions. It is a member of a growing family that includes several structurally distinct protein tyrosine kinases, such as related adhesion focal tyrosine kinase and calcium-dependent protein tyrosine kinase (6). FAK can be activated by specific extracellular stimuli, such as integrins and certain growth factors (7). FAK is encoded by the protein tyrosine kinase 2 (PTK2) gene, which is located at human chromosome region $8 \mathrm{q} 24.3$. It has been found that the region is commonly amplified in several types of cancer, such as serous ovarian (8) 
and gastric (9) cancer. Abnormally increased FAK expression has additionally been found in several types of cancer (10), such as cervical (11), ovarian (12), breast (13) and lung (14) cancer. FAK plays an important role in signal transduction in the tumor microenvironment (15), as it is a multifunctional scaffolding molecule that links transmembrane input signals from growth factor receptors and integrins to intracellular effectors, such as c-Jun N-terminal kinase and phosphatidylinositol 3-kinase/Akt (PI3K/Akt) (16). FAK proteins are known to regulate cell survival and apoptosis via several pathways, such as PI3K/Akt (17).

An increased FAK mRNA level is found in bladder cancer (10); however, no study to date has been performed to determine whether FAK is associated with the survival and apoptosis of bladder cancer cells. The aim of the present study, therefore, was to explore the potential role of FAK in the apoptosis of bladder cancer cells.

\section{Materials and methods}

Cell culture and reagents. The T24 human bladder cancer cell line was purchased from the American Type Culture Collection (Manassas, VA, USA). The cells were cultured in Dulbecco's modified Eagle's medium (Gibco-BRL, Grand Island, NY, USA), supplemented with $10 \%$ fetal bovine serum at $37^{\circ} \mathrm{C}$ in a humidified $5 \% \mathrm{CO}_{2} / 95 \%$ air atmosphere. The FAK inhibitor PF-573228 (PF-228), the Src inhibitor PP2 and the PI3K inhibitor LY294002 were obtained from Sigma-Aldrich (St. Louis, MO, USA). TGF $\beta$ was obtained from Peprotech, Inc. (Rocky Hill, NJ, USA).

Preparation and transfection of small interfering RNAs (siRNAs). siRNAs against FAK [sense, 5'-UAAUACUCGCUC CAUUGCACC(dT)(dT)-3' and antisense, 5'-GGUGCAAUG GAGCGAGUAUUA(dT)(dT)-3'] were designed as described previously (18). The siRNA duplexes were chemically synthesized by GeneChem Co., Ltd. (Shanghai, China). Transfections were performed in six-well plates (Corning Costar, Cambridge, MA, USA) with Lipofectamine ${ }^{\mathrm{TM}} 2000$ transfection reagent (Invitrogen Life Technologies, Carlsbad, CA, USA) according to the manufacturer's instructions.

Quantitative polymerase chain reaction ( $q P C R)$. The treated cells were collected and the total RNA was prepared using TRIzol ${ }^{\circledR}$ reagent (Invitrogen Life Technologies). Subsequently, reverse transcription was performed with $2 \mu \mathrm{g}$ total RNA using a one-step RT-PCR system (Invitrogen Life Technologies). qPCR was conducted in an Applied Biosystems 7300 Realtime PCR Instrument (Applied Biosystems Life Technologies, Foster City, CA, USA). The PCR cycling conditions were as follows: $95^{\circ} \mathrm{C}$ for $3 \mathrm{~min}$, followed by cycles at $95^{\circ} \mathrm{C}$ for $10 \mathrm{sec}$ and $60^{\circ} \mathrm{C}$ for $20 \mathrm{sec}$, then $72^{\circ} \mathrm{C}$ for $15 \mathrm{sec}$. The PCR products were detected using SYBR ${ }^{\circledR}$ Green dye (Applied Biosystems Life Technologies) according to the manufacturer's instructions. Specific oligonucleotide primers were synthesized by Sangon Biotech Co., Ltd. (Shanghai, China). Relative quantification of the mRNA levels of target genes was performed using the $2^{-\Delta \Delta C T}$ method, as described previously (19).

Western blot analysis. Western blot analysis was performed as described previously (20). Total protein $(50 \mu \mathrm{g})$ from each sample was loaded for SDS-PAGE. The membrane was exposed on an X-ray film (Eastman Kodak Co., Rochester, NY, USA) using enhanced chemiluminescence western blot detection reagents (Pierce Biotechnology, Inc., Rockford, IL, USA). Cumulative gray levels of all bands were calculated using ImageJ software (National Institutes of Health, Bethesda, MD, USA) for further relative quantitative analysis. Primary antibodies that were specific against following proteins were used: Rabbit polyclonal FAK (\#3285; 1:1,000), rabbit polyclonal phosphorylated (p)FAK (\#3283; 1:1,000), rabbit mAb Src (\#2109; 1:1,000), rabbit polyclonal pSrc (\#2101; 1:1,000), mouse mAb Akt (\#2966; 1:500), mouse mAb pAkt (\#4051; 1:1,000), mouse mAb caspase-3 (\#9668; 1:1,000) and cleaved caspase-3 (\#9579; 1:1,000) (all Cell Signaling Technology, Inc., Danvers, MA, USA) and rabbit polyclonal $\beta$-actin (sc-1616-R; 1:2,000; Santa Cruz Biotechnology, Inc., Santa Cruz, CA, USA). The membrane was incubated with primary antibodies at $4^{\circ} \mathrm{C}$ overnight. Horseradish peroxidase-labeled secondary antibodies were obtained from Santa Cruz Biotechnology, Inc.

In situ deoxynucleotidyl transferase-mediated dUTP-biotin nick end labeling assay. In situ TUNEL assay was employed to detect the apoptotic cells. T24 cells were grown on slides and fixed with $4 \%$ buffered formaldehyde. TUNEL assay was performed using the in situ Cell Death Detection kit (Boehringer Mannheim GmbH, Mannheim, Germany) as described previously (18). The slides were counterstained with hematoxylin. The apoptotic cells were stained brown under the microscope.

Annexin $V$ labeling. To measure the numbers and the ratio of apoptotic cells, the Annexin V-fluorescein isothiocyanate (FITC) Apoptosis Detection kit (BD Biosciences, Franklin Lakes, NJ, USA) was employed as described previously (18). The treated cells were stained with FITC, Annexin V and propidium iodide (PI), and the stained cells were analyzed using a FACSort ${ }^{\mathrm{TM}}$ flow cytometer (Becton-Dickinson, Franklin Lakes, NJ, USA) and evaluated with the CellQuest ${ }^{\mathrm{TM}}$ software system (BD Biosciences).

Cell viability assay. Cell viability was determined using the MTT assay as described previously (18). Cells were cultured in 96 -well plates at a density of $2 \times 10^{4}$ cells/well. The cell viability was measured using the MTT assay. Cells were incubated with $10 \mu 10.5 \mathrm{mg} / \mathrm{ml} \mathrm{MTT}$ at $37^{\circ} \mathrm{C}$ for $4 \mathrm{~h}$. The formazan crystals were dissolved using $200 \mu \mathrm{l}$ dimethylsulfoxide and quantified by measuring absorbance at $570 \mathrm{~nm}$.

Statistical analysis. All experiments were repeated at least three times, and the data were analyzed using the SPSS 12.0 statistical software package (SPSS, Inc., Chicago, IL, USA). One-way analysis of variance and the Student's $t$-test were employed to compare the data. $\mathrm{P}<0.05$ was considered to indicate a statistically significant difference.

\section{Results}

Knockdown of FAK induces apoptosis in T24 bladder cancer cells. To identify whether FAK affected the survival or apoptosis of T24 bladder cancer cells, FAK expression in 


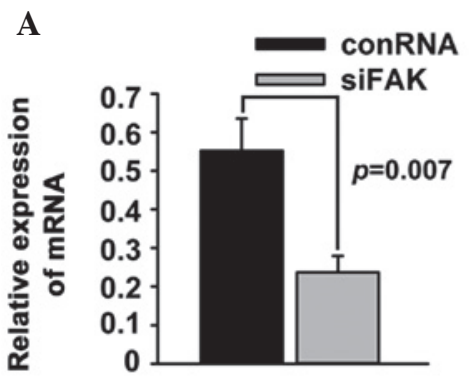

B

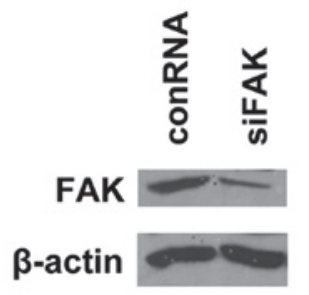

C
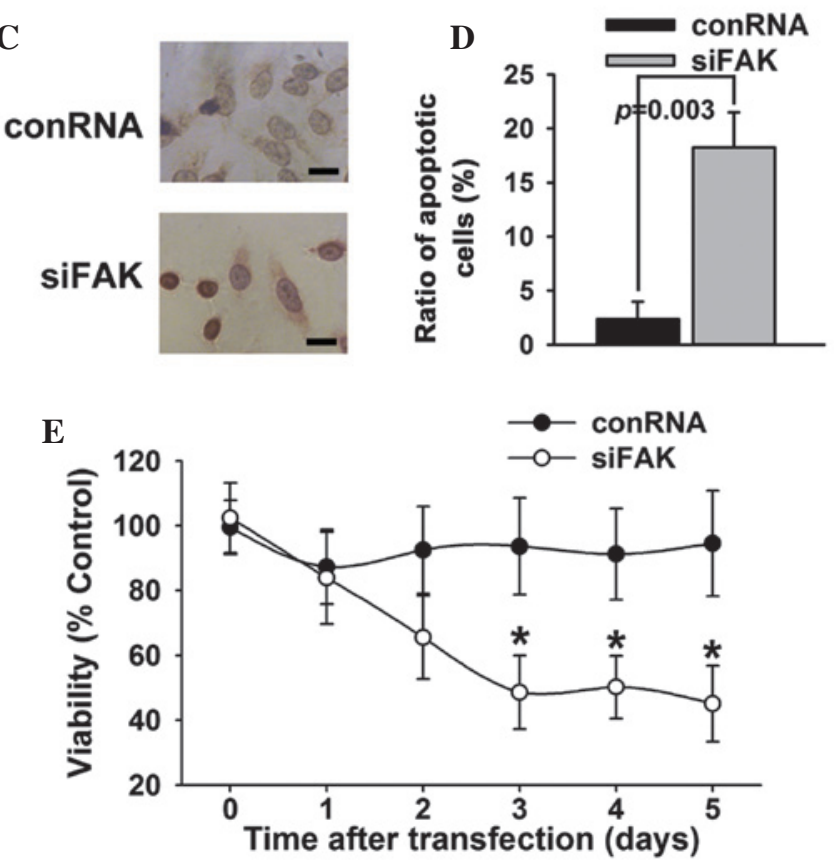

Figure 1. Knockdown of FAK induces apoptosis in T24 bladder cancer cells. T24 bladder cancer cells were transfected with small interfering RNA against FAK (siFAK) or control (conRNA). (A and B) The expression of FAK was examined using western blotting. (C and D) Cell apoptosis was examined using (C) deoxynucleotidyl transferase-mediated dUTP-biotin nick end labeling assay and (D) Annexin V/propidium iodide. (E) Cell survival was examined using an MTT assay. Scale bar, $200 \mu \mathrm{m}$. ${ }^{*} \mathrm{P}<0.05$. FAK, focal adhesion kinase.

T24 bladder cancer cells was knocked down using a specific siRNA duplex targeting FAK. As shown in Fig. 1A and B, the siRNA duplex caused a marked decrease of FAK mRNA and protein expression in the T24 bladder cancer cells. The results of TUNEL (Fig. 1C) and Annexin V/PI (Fig. 1D) assays showed that siRNA against FAK significantly increased the apoptosis of T24 bladder cancer cells. The results of the MTT assays (Fig. 1E) showed that inhibition of FAK expression by siRNA decreased the viability of the T24 cells compared with control RNA.

Suppression of FAK phosphorylation induces apoptosis in T24 bladder cancer cells. To further investigate whether the inhibition of FAK tyrosine phosphorylation could promote the apoptosis of T24 bladder cancer cells, PF-228, a selective inhibitor of FAK, was employed to inhibit the transforming growth factor- $\beta$ (TGF $\beta$ )-induced tyrosine phosphorylation of FAK. The results of the TUNEL (Fig. 2A) and Annexin V/PI (Fig. 2B) assays showed that PF-228 was able to induce the apoptosis of T24 bladder cancer cells. The western blotting results
A
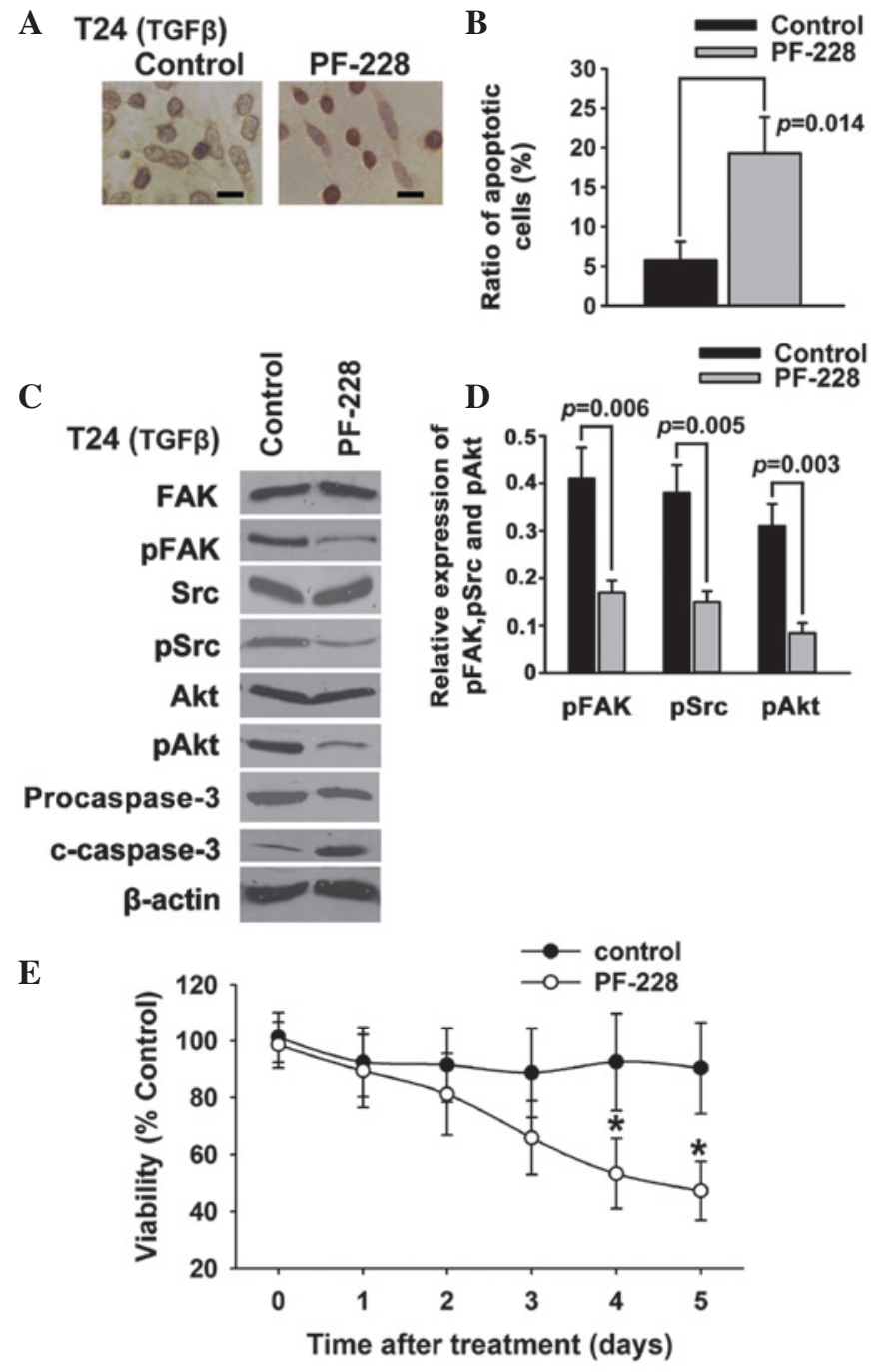

Figure 2. Suppression of FAK phosphorylation induces apoptosis in T24 bladder cancer cells. T24 bladder cancer cells were treated with PF-228 and $5 \mathrm{ng} / \mathrm{ml} \mathrm{TGF} \beta$. (A and B) Cell apoptosis was examined using (A) deoxynucleotidyl transferase-mediated dUTP-biotin nick end labeling assay and (B) Annexin V/propidium iodide. (C and D) The expression of FAK, pFAK, Src, pSrc, Akt, pAkt, caspase-3 and c-caspase-3 was examined using western blotting. (E) Cell survival was examined using an MTT assay. Scale bar, $200 \mu \mathrm{m}$. ${ }^{*} \mathrm{P}<0.05$. FAK, focal adhesion kinase; pFAK, phosphorylated FAK; c-caspase-3, cleaved caspase- 3 ; TGF $\beta$, transforming growth factor- $\beta$.

indicated that PF-228 significantly reduced the TGF $\beta$-induced phosphorylation of FAK and Src, suppressed the phosphorylation of Akt, an accepted signal of cell survival/apoptosis, and activated caspase-3, an important apoptosis-related protein (Fig. 2C and D). The results of the MTT assays (Fig. 2E) showed that inhibition of FAK by PF-228 decreased the viability of the T24 cells compared with the control.

Src is an important mediator of FAK-regulated apoptosis in T24 bladder cancer cells. FAK is a substrate for the oncogene protein tyrosine kinase Src (21). To further whether the inhibition of Src phosphorylation could also promote the apoptosis of T24 bladder cancer cells, PP2, a selective inhibitor of Src, was employed to inhibit the TGF $\beta$-induced phosphorylation of Src. The results of the TUNEL (Fig. 3A) and Annexin V/PI (Fig. 3B) assays showed that PP2, similarly to the FAK inhib- 

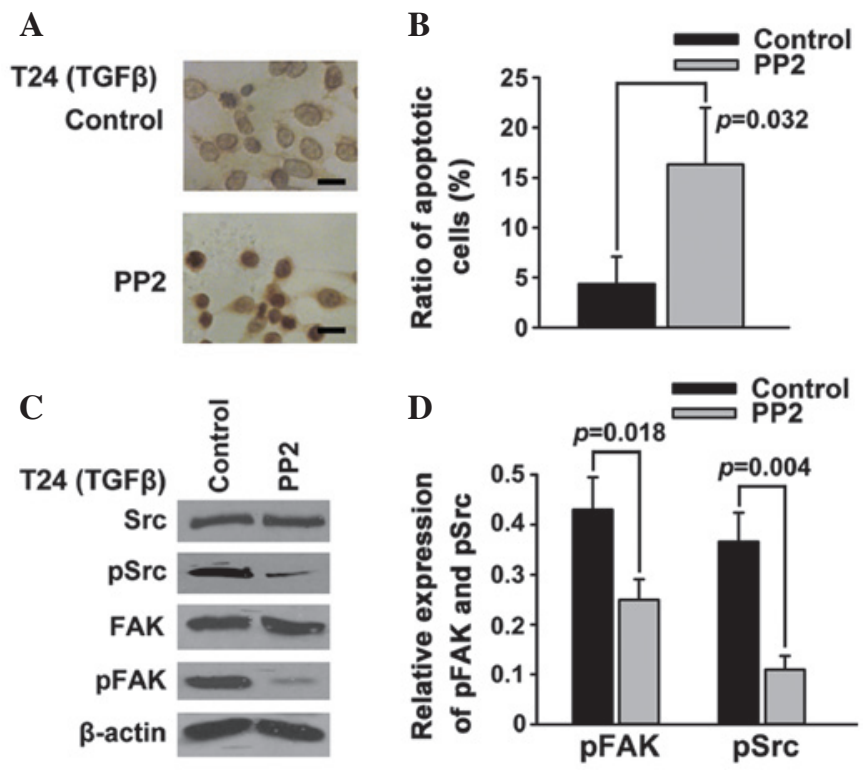

Figure 3. Src is an important mediator of FAK-regulated apoptosis in T24 bladder cancer cells. T24 bladder cancer cells were treated with PP2. (A and B) Cell apoptosis was examined using (A) deoxynucleotidyl transferase-mediated dUTP-biotin nick end labeling assay and (B) Annexin V/propidium iodide. (C and D) The expression of FAK, pFAK, Src and pSrc was examined using western blotting. Scale bar, $200 \mu \mathrm{m}$. FAK, focal adhesion kinase; pFAK, phosphorylated FAK; TGF $\beta$, transforming growth factor- $\beta$.

itor PF-228, was able to induce the apoptosis of T24 bladder cancer cells. The western blotting results demonstrated that PP2 significantly reduced the TGF $\beta$-induced phosphorylation of not only Src but also FAK (Fig. 3C and D).

PI3K/Akt signaling acts downstream of FAK to regulate apoptosis in T24 bladder cancer cells. PI3K/Akt is a potent pathway of survival and apoptosis (22), and FAK is believed to be an upstream signal protein of the PI3K/Akt pathway (23); therefore, it was investigated whether PI3K/Akt acted downstream of FAK to regulate the apoptosis of T24 bladder cancer cells. Western blot analysis showed that Akt and FAK phosphorylation was suppressed by PF-228 (Fig. 2C and D). In addition, LY294002 significantly downregulated pAkt in the T24 cells (Fig. 4A and B). The results of the TUNEL (Fig. 4C) and Annexin V/PI (Fig. 4D) assays showed that LY294002 was able to induce the apoptosis of T24 cells. Conversely, the expression of general and tyrosine-phosphorylated FAK was not regulated by inhibiting PI3K/Akt in T24 cells (Fig. 4E and F).

\section{Discussion}

FAK was first described by Linder and Burr in 1988 as a $120-\mathrm{kDa}$ protein that was one of a large number of tyrosine phosphoproteins in Rous sarcoma virus-transformed chicken embryo fibroblasts (24). Reynolds et al (25) reported the finding of a $120-\mathrm{kDa}$ protein whose phosphorylation was greatly enhanced in cells expressing activated, oncogenic Src in 1989, and the same research group generated monoclonal antibodies against the 120-kDa protein in 1990 (26). Furthermore, a $120-\mathrm{kDa}$ protein was described to tyrosine-phosphorylate in

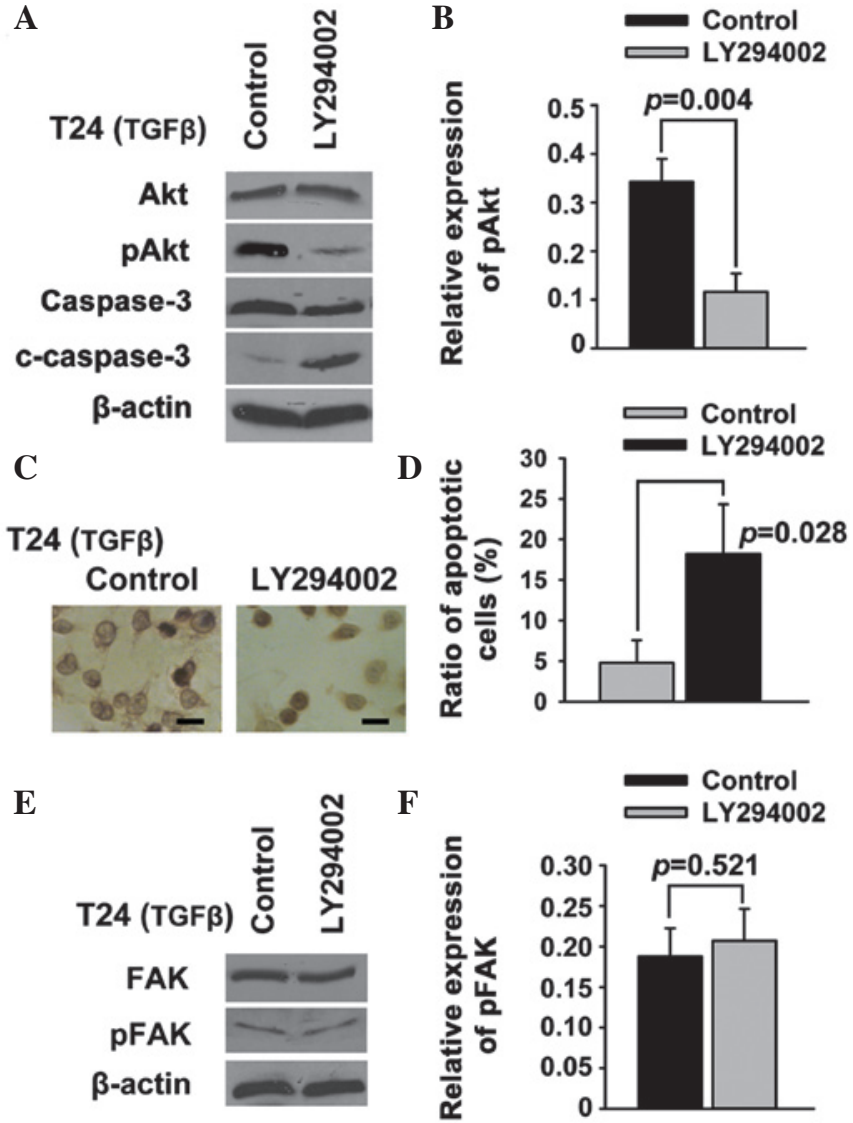

Figure 4. Phosphatidylinositol 3-kinase/Akt acts downstream of FAK signaling to regulate apoptosis in T24 bladder cancer cells. T24 bladder cancer cells were treated with LY294002. (A and B) The expression of Akt, pAkt, caspase-3 and c-caspase-3 was examined using western blotting. (C and D) Cell apoptosis was examined using (C) deoxynucleotidyl transferase-mediated dUTP-biotin nick end labeling assay and (D) Annexin V/propidium iodide. (E and F) The expression of FAK and pFAK was examined using western blotting. Scale bar, $200 \mu \mathrm{m}$. FAK, focal adhesion kinase; pFAK, phosphorylated FAK; c-caspase-3, cleaved caspase-3; TGF $\beta$, transforming growth factor- $\beta$.

fibronectin-stimulated cells by Guan et al in 1991 (27). The protein was located in focal contacts, where it codistributed with $\beta 1$ integrins. Phosphorylation of the protein was correlated with subsequent cell spreading. It was suggested that the interaction of $\beta 1$ integrins with extracellular ligands, such as fibronectin, turned on the phosphorylation of the $120-\mathrm{kDa}$ protein, which may have been involved in the responses of cells to attachment. Schaller et al (28) identified a $125-\mathrm{kDa}$ phosphotyrosine-containing protein as a tyrosine phosphatase substrate of v-Src in chicken embryo cells in 1992. In the study by Schaller et al, cDNA of the protein was isolated, and the predicted structure of the new protein, which was the prototype for an additional family of protein-tyrosine kinases, was found. Since the protein was localized to focal adhesions, they named the new protein focal adhesion kinase. In the same year, Hanks et al (29) found that the activation of FAK via tyrosine phosphorylation was an important early step in intracellular signal transduction pathways in response to extracellular stimuli with the extracellular matrix. In 1994, Schaller et al (30) suggested that Tyr-397 was a major site of FAK autophosphorylation and that FAK was physically associated with Src via their SH2 domains. More recent research has shown that FAK links extracellular stimuli, such as integrins 
and growth factors, to intracellular signaling and regulates cell movement, migration, invasion, survival and cancer stem cell self-renewal $(10,31)$. The aim of the present study was to determine if FAK regulated the survival and apoptosis of bladder cancer cells.

Apoptosis is the process of programmed cell death that may occur in multicellular organisms and is characterized by morphological changes of the cells, include blebbing, cell shrinkage, nuclear fragmentation, chromatin condensation and chromosomal DNA fragmentation (32). The activation of FAK by the interaction of extracellular matrix signals and integrins has been shown to be accompanied by suppressed apoptosis in diverse cell types (33). Notably, FAK was found to be tyrosine-phosphorylated by oxidative stress prior to the occurrence of apoptosis (34). Sonoda et al (34) found that FAK retained tyrosine phosphorylation at least up to $5 \mathrm{~h}$ and gradually lost tyrosine phosphorylation after $8 \mathrm{~h}$, concomitant with apoptosis. While FAK was inhibited by inhibitor of protein tyrosine kinases or antisense oligonucleotide against FAK, apoptosis was accelerated. Sonoda et al suggested that tyrosine phosphorylation of FAK played a suppressive role in cell apoptosis. Since it has been shown that specific siRNAs targeting mRNA of the PTK2 gene are effective in inhibiting the expression of FAK (35-39), RNA interference was employed in the present study as a potent tool to explore the role of FAK in the survival and apoptosis of bladder cancer cells. The results showed that apoptosis was induced in T24 bladder cancer cells when FAK was suppressed by siRNA targeting FAK. Similar results were observed following the administration of PF-228, an exclusive inhibitor of FAK tyrosine phosphorylation. These results suggest that not only the general expression but also the tyrosine phosphorylation level of FAK is associated with the apoptosis of bladder cancer cells.

FAK is an important mediator of TGF $\beta$ signaling (40). TGF $\beta$ predominantly regulates FAK via tyrosine phosphorylation of FAK (41). FAK was first described as a $120-\mathrm{kDa}$ protein that was tyrosine-phosphorylated following induction by Src (24). FAK is autophosphorylated at Tyr-397 due to input signals from integrins and growth factor receptors, to which Src proteins subsequently bind. Afterwards, FAK-Src complexes are formed, and FAK is phosphorylated at Tyr-576 and Tyr-577. The complexes play an important role in mediating the extracellular signal to downstream molecules such as extracellular signal-regulated kinases and paxillin $(42,43)$; therefore, Src is one of the most important regulatory proteins in FAK-related signals. PP2 is a selective inhibitor of Src family members and is able to block the Tyr-416 phosphorylation of Src (44). In the present study, therefore, the effect of Src tyrosine dephosphorylation on FAK and the apoptosis of bladder cancer cells was explored using PP2. The results showed that PP2 was able to induce the apoptosis of T24 cells, while tyrosine phosphorylation of not only Src but also FAK was inhibited in TGF $\beta$-stimulated bladder cancer cells.

Notably, Wen et al (45) found that FAK is cleaved into two different fragments in early apoptosis, which is mediated by caspase-7 and caspase-3 (45). They suggested that the disruption of FAK may contribute to the morphological changes of cells in apoptosis. Levkau et al (46) found that cleavage of FAK affected its association with signaling and other cytoskeletal components of the focal adhesion complex, such as paxillin. They suggested that the caspase-mediated cleavage of FAK disturbed survival signals from the extracellular matrix and propagated the cell death program. Van de Water et al (47) found that the inhibition of caspase activity blocked FAK cleavage and apoptosis, but not FAK dephosphorylation. They suggested that caspases are required for FAK cleavage, but not for FAK dephosphorylation, during apoptosis. Sonoda et al (23) demonstrated that the tyrosine phosphorylation of FAK, the association of FAK with PI3K and the serine phosphorylation of Akt occurred during oxidative stress-induced apoptosis. They suggested that FAK was an upstream signal protein of the PI3K/Akt pathway during oxidative stress-induced apoptosis. In another study, Sonoda et al (48) found that the PI3K/Akt survival pathway was activated, and the activation of procaspase-3 to caspase-3 was inhibited, in FAK-transfected cells, which had resistance to apoptotic stimuli. They suggested that FAK activated the PI3K/Akt survival pathway, as well as its downstream signals, and finally inhibited apoptosis by blocking the caspase-3 cascade. The role of the PI3K/Akt pathway and caspase- 3 during the apoptosis of bladder cancer cells induced by inhibiting FAK was therefore explored in the present study. The results showed that the phosphorylation of Akt was suppressed and the activation of procaspase-3 to caspase-3 was induced during apoptosis, while FAK was dephosphorylated by a tyrosine phosphorylation inhibitor. LY294002, a common inhibitor of PI3K, was additionally utilized to investigate the effect of inhibiting the PI3K/Akt pathway on the general expression and tyrosine phosphorylation level of FAK. The results showed that inhibiting the PI3K/Akt pathway was able to induce the apoptosis of T24 cells, but did not regulate either the general expression or the tyrosine phosphorylation of FAK. These results suggested that PI3K/Akt acted downstream of FAK signaling to regulate apoptosis in bladder cancer cells.

In this study, the regulatory role of FAK signaling on the apoptosis and survival of bladder cancer cells was demonstrated. Both the knockdown of FAK and the suppression of FAK phosphorylation were able to induce apoptosis in bladder cancer cells. Caspase-3 was activated during the apoptosis induced by the suppression of FAK phosphorylation. Src was involved in FAK-regulated apoptosis in bladder cancer cells, while the suppression of Src phosphorylation was able to inhibit FAK tyrosine phosphorylation and induce apoptosis. Furthermore, PI3K/Akt signaling was inhibited via the suppression of FAK tyrosine phosphorylation. Conversely, neither the expression of general nor tyrosine-phosphorylated FAK was regulated by inhibiting PI3K/Akt. These results suggested that PI3K/Akt acted downstream of FAK signaling to regulate apoptosis in bladder cancer cells. Collectively, the data indicate that FAK is an important regulator of apoptosis and survival signaling in bladder cancer cells.

\section{Acknowledgements}

The present study was supported by the National Natural Science Foundation of China (nos. 30901499 and 81272862), the Zhejiang Provincial Natural Science Foundation of China (nos. LY12H16020 and LY13H160020) and the Zhejiang Provincial Medical Science and Technology Program (nos. 2013RCA014 and 2014KYA117). 


\section{References}

1. Torre LA, Bray F, Siegel RL, Ferlay J, Lortet-Tieulent J and Jemal A: Global cancer statistics, 2012. CA Cancer J Clin 65: $87-108,2015$

2. Morgan TM, Keegan KA and Clark PE: Bladder cancer. Curr Opin Oncol 23: 275-282, 2011

3. van Rhijn BW, Burger M, Lotan Y, Solsona E, Stief CG, Sylvester RJ, Witjes JA and Zlotta AR: Recurrence and progression of disease in non-muscle-invasive bladder cancer: From epidemiology to treatment strategy. Eur Urol 56: 430-442, 2009.

4. Lee CT, Dunn RL, Ingold C, Montie JE and Wood DP Jr: Early-stage bladder cancer surveillance does not improve survival if high-risk patients are permitted to progress to muscle invasion. Urology 69: 1068-1072, 2007.

5. Prout GR Jr, Griffin PP and Shipley WU: Bladder carcinoma as a systemic disease. Cancer 43: 2532-2539, 1979.

6. Parsons JT: Focal adhesion kinase: The first ten years. J Cell Sci 116: 1409-1416, 2003.

7. Lechertier T and Hodivala-Dilke K: Focal adhesion kinase and tumour angiogenesis. J Pathol 226: 404-412, 2012.

8. Okamoto H, Yasui K, Zhao C, Arii S and Inazawa J: PTK2 and EIF3S3 genes may be amplification targets at 8q23-q24 and are associated with large hepatocellular carcinomas. Hepatology 38 1242-1249, 2003.

9. Park JH, Lee BL, Yoon J, Kim J, Kim MA, Yang HK and Kim WH: Focal adhesion kinase (FAK) gene amplification and its clinical implications in gastric cancer. Hum Pathol 41: 1664-1673, 2010.

10. Sulzmaier FJ, Jean C and Schlaepfer DD: FAK in cancer: Mechanistic findings and clinical applications. Nat Rev Cancer 14: 598-610, 2014.

11. Oktay MH, Oktay K, Hamele-Bena D, Buyuk A and Koss LG: Focal adhesion kinase as a marker of malignant phenotype in breast and cervical carcinomas. Hum Pathol 34: 240-245, 2003

12. Cancer Genome Atlas Research Network: Integrated genomic analyses of ovarian carcinoma. Nature 474: 609-615, 2011.

13. Cancer Genome Atlas N Network: Comprehensive molecular portraits of human breast tumours. Nature 490: 61-70, 2012.

14. Lu H, Wang L, Gao W, Meng J, Dai B, Wu S, Minna J, Roth JA, Hofstetter WL, Swisher SG and Fang B: IGFBP2/FAK pathway is causally associated with dasatinib resistance in non-small cell lung cancer cells. Mol Cancer Ther 12: 2864-2873, 2013.

15. Schober M and Fuchs E: Tumor-initiating stem cells of squamous cell carcinomas and their control by TGF- $\beta$ and integrin/focal adhesion kinase (FAK) signaling. Proc Natl Acad Sci USA 108: 10544-10549, 2011.

16. Wendt MK, Smith JA and Schiemann WP: Transforming growth factor- $\beta$-induced epithelial-mesenchymal transition facilitates epidermal growth factor-dependent breast cancer progression. Oncogene 29: 6485-6498, 2010.

17. Luo M and Guan JL: Focal adhesion kinase: A prominent determinant in breast cancer initiation, progression and metastasis. Cancer Lett 289: 127-139, 2010.

18. Sima N, Wang W, Kong D, Deng D, Xu Q, Zhou J, Xu G, Meng L, Lu Y, Wang S and Ma D: RNA interference against HPV16 E7 oncogene leads to viral E6 and E7 suppression in cervical cancer cells and apoptosis via upregulation of $\mathrm{Rb}$ and $\mathrm{p} 53$. Apoptosis 13 : 273-281, 2008

19. Li BH, Zhou JS, Ye F, Cheng XD, Zhou CY, Lu WG and Xie X: Reduced miR-100 expression in cervical cancer and precursors and its carcinogenic effect through targeting PLK1 protein. Eur J Cancer 47: 2166-2174, 2011.

20. Sima N, Wang S, Wang W, Kong D, Xu Q, Tian X, Luo A, Zhou J, $\mathrm{Xu} \mathrm{G}$, Meng L, et al: Antisense targeting human papillomavirus type 16 E6 and E7 genes contributes to apoptosis and senescence in SiHa cervical carcinoma cells. Gynecol Oncol 106: 299-304, 2007.

21. Guan JL and Shalloway D: Regulation of focal adhesion-associated protein tyrosine kinase by both cellular adhesion and oncogenic transformation. Nature 358: 6388, 1992.

22. Fresno Vara JA, Casado E, de Castro J, Cejas P, Belda-Iniesta C and González-Barón M: PI3K/Akt signalling pathway and cancer. Cancer Treat Rev 30: 193-204, 2004.

23. Sonoda Y, Watanabe S, Matsumoto Y, Aizu-Yokota E and Kasahara T: FAK is the upstream signal protein of the phosphatidylinositol 3-kinase-Akt survival pathway in hydrogen peroxide-induced apoptosis of a human glioblastoma cell line. J Biol Chem 274: 10566-10570, 1999.
24. Linder ME and Burr JG: Nonmyristoylated p60v-src fails to phosphorylate proteins of $115-120 \mathrm{kDa}$ in chicken embryo fibroblasts. Proc Natl Acad Sci USA 85: 2608-2612, 1988.

25. Reynolds AB, Roesel DJ, Kanner SB and Parsons JT: Transformation-specific tyrosine phosphorylation of a novel cellular protein in chicken cells expressing oncogenic variants of the avian cellular src gene. Mol Cell Biol 9: 629-638, 1989.

26. Kanner SB, Reynolds AB, Vines RR and Parsons JT: Monoclonal antibodies to individual tyrosine-phosphorylated protein substrates of oncogene-encoded tyrosine kinases. Proc Natl Acad Sci USA 87: 3328-3332, 1990.

27. Guan JL, Trevithick JE and Hynes RO: Fibronectin/integrin interaction induces tyrosine phosphorylation of a $120-\mathrm{kDa}$ protein. Cell Regul 2: 951-964, 1991.

28. Schaller MD, Borgman CA, Cobb BS, Vines RR, Reynolds AB and Parsons JT: pp125FAK a structurally distinctive protein-tyrosine kinase associated with focal adhesions. Proc Natl Acad Sci USA 89: 5192-5196, 1992.

29. Hanks SK, Calalb MB, Harper MC and Patel SK: Focal adhesion protein-tyrosine kinase phosphorylated in response to cell attachment to fibronectin. Proc Natl Acad Sci USA 89: 8487-8491, 1992.

30. Schaller MD, Hildebrand JD, Shannon JD, Fox JW, Vines RR and Parsons JT: Autophosphorylation of the focal adhesion kinase, pp125FAK, directs SH2-dependent binding of pp60src. Mol Cell Biol 14: 1680-1688, 1994.

31. Park MS, Kim YH and Lee JW: FAK mediates signal crosstalk between type II collagen and TGF-beta 1 cascades in chondrocytic cells. Matrix Biol 29: 135-142, 2010.

32. Duvall E, Wyllie AH and Morris RG: Macrophage recognition of cells undergoing programmed cell death (apoptosis). Immunology 56: 351-358, 1985.

33. Frisch SM, Vuori K, Ruoslahti E and Chan-Hui PY: Control of adhesion-dependent cell survival by focal adhesion kinase. J Cell Biol 134: 793-799, 1996.

34. Sonoda Y, Kasahara T, Yokota-Aizu E, Ueno M and Watanabe S: A suppressive role of $\mathrm{p} 125 \mathrm{FAK}$ protein tyrosine kinase in hydrogen peroxide-induced apoptosis of T98G cells. Biochem Biophys Res Commun 241: 769-774, 1997.

35. Clemente CF, Tornatore TF, Theizen TH, Deckmann AC, Pereira TC, Lopes-Cendes I, Souza JR and Franchini KG: Targeting focal adhesion kinase with small interfering RNA prevents and reverses load-induced cardiac hypertrophy in mice. Circ Res 101: 1339-1348, 2007.

36. Duxbury MS, Ito H, Benoit E, Zinner MJ, Ashley SW and Whang EE: RNA interference targeting focal adhesion kinase enhances pancreatic adenocarcinoma gemcitabine chemosensitivity. Biochem Biophys Res Commun 311: 786-792, 2003.

37. Chan KT, Cortesio CL and Huttenlocher A: FAK alters invadopodia and focal adhesion composition and dynamics to regulate breast cancer invasion. J Cell Biol 185: 357-370, 2009.

38. Thamilselvan V, Craig DH and Basson MD: FAK association with multiple signal proteins mediates pressure-induced colon cancer cell adhesion via a Src-dependent PI3K/Akt pathway. FASEB J 21: 1730-1741, 2007.

39. Shi Q, Bao S, Song L, Wu Q, Bigner DD, Hjelmeland AB and Rich JN: Targeting SPARC expression decreases glioma cellular survival and invasion associated with reduced activities of FAK and ILK kinases. Oncogene 26: 4084-4094, 2007.

40. Leask A: Focal adhesion kinase: A key mediator of transforming growth factor beta signaling in fibroblasts. Adv Wound Care (New Rochelle) 2: 247-249, 2013.

41. Wang SE, Xiang B, Zent R, Quaranta V, Pozzi A and Arteaga CL: Transforming growth factor beta induces clustering of HER 2 and integrins by activating Src-focal adhesion kinase and receptor association to the cytoskeleton. Cancer Res 69: 475-482, 2009.

42. Schaller MD: Cellular functions of FAK kinases: Insight into molecular mechanisms and novel functions. J Cell Sci 123: 1007-1013, 2010.

43. Mitra SK and Schlaepfer DD: Integrin-regulated FAK-Src signaling in normal and cancer cells. Curr Opin Cell Biol 18: 516-523, 2006.

44. Chiang GJ, Billmeyer BR, Canes D, Stoffel J, Moinzadeh A, Austin CA, Kosakowski M, Rieger-Christ KM, Libertino JA and Summerhayes IC: The src-family kinase inhibitor PP2 suppresses the in vitro invasive phenotype of bladder carcinoma cells via modulation of Akt. BJU Int 96: 416-422, 2005.

45. Wen LP, Fahrni JA, Troie S, Guan JL, Orth K and Rosen GD: Cleavage of focal adhesion kinase by caspases during apoptosis. J Biol Chem 272: 26056-26061, 1997. 
46. Levkau B, Herren B, Koyama H, Ross R and Raines EW: Caspase-mediated cleavage of focal adhesion kinase pp125FAK and disassembly of focal adhesions in human endothelial cell apoptosis. J Exp Med 187: 579-586, 1998.

47. van de Water B, Nagelkerke JF and Stevens JL: Dephosphorylation of focal adhesion kinase (FAK) and loss of focal contacts precede caspase-mediated cleavage of FAK during apoptosis in renal epithelial cells. J Biol Chem 274: 13328-13337, 1999.
48. Sonoda Y, Matsumoto Y, Funakoshi M, Yamamoto D, Hanks SK and Kasahara T: Anti-apoptotic role of focal adhesion kinase (FAK). Induction of inhibitor-of-apoptosis proteins and apoptosis suppression by the overexpression of FAK in a human leukemic cell line, HL-60. J Biol Chem 275: $16309-16315,2000$ 